\title{
Possibility of repeat surgery for recurrence following two-stage hepatectomy for colorectal liver metastases: impact on patient outcome
}

\author{
Francesco Ardito ${ }^{1,2}$ \\ ${ }^{1}$ Hepatobiliary Surgery Unit, Fondazione Policlinico Universitario A. Gemelli IRCCS; Rome, Italy; ${ }^{2}$ Institute of Surgical Pathology, Università \\ Cattolica del Sacro Cuore, Rome, Italy \\ Correspondence to: Francesco Ardito, MD, PhD. Hepato-Biliary Surgery Unit, Fondazione Policlinico Universitario A. Gemelli IRCCS, Largo \\ Agostino Gemelli, 8 - I-00168 Rome, Italy, Email: francesco.ardito@unicatt.it.
}

Submitted Jul 22, 2019. Accepted for publication Sep 11, 2019.

doi: 10.21037/hbsn.2019.09.08

View this article at: http://dx.doi.org/10.21037/hbsn.2019.09.08

Hepatic resection together with perioperative chemotherapy is currently the only treatment option that can offer a chance of long-term outcome in patients with colorectal liver metastases (CRLM), resulting in 5-year survival rates of $40 \%$, and exceeding $50 \%$ in selected patients $(1,2)$.

The safety of liver resections in high-volume centers and the increased efficacy of perioperative chemotherapy regimens have improved and expanded the indications to surgery for CRLM (3). Patients with advanced disease (extensive multinodular and bilateral CRLM), often classified as unresectable at the time of diagnosis have the possibility to undergo curative liver resection after downstaging by conversion chemotherapy $(4,5)$. In such patients, the multilocularity and bilaterality of the disease may represent a technical issue for liver resection because of the inability to achieve radical resection while preserving sufficient functional liver volume.

Surgical management for patients with advanced bilateral CRLM includes three main strategies: the two-stage hepatectomy (TSH), initially described by Adam et al. in 2000 (6), the enhanced one-stage parenchymal-sparing hepatectomy, described by Torzilli et al. (7), and the associating liver partition and portal vein ligation for staged hepatectomy (ALPPS) procedure described by Schnitzbauer et al. (8).

By the use of these surgical techniques, an increasing number of patients with advanced bilobar CRLM have undergone curative surgical resection with 5 -year overall survival rates similar to those following resections for unilobar disease (9).

However, despite the recent improved overall survival of patients resected for CRLM, recurrence still represents the critical oncological issue. Indeed about $60-70 \%$ of resected patients will recur and more than $50 \%$ of patients will develop recurrence within 2 years after liver resection (10). Of course, the risk of liver recurrence following aggressive procedures such as TSH, enhanced one-stage hepatectomy and ALPPS, is reported to be higher because of the more extensive disease in such patients. Indeed, the rate of recurrence following TSH is reported to be $>70 \%(11,12)$.

It has been largely shown that repeat hepatectomy for liver recurrence is safe and effective in selected patients (13). Indeed, repeat hepatectomy may increase the chance of long-term survival for patients with recurrence in the liver, with reported 5 -year survival rates ranging from $30 \%$ to $60 \%$. These survival rates are comparable to those of patients who only underwent single hepatectomy.

Because of the high risk of liver recurrence following liver resection for CRLM, the first liver resection should always be performed taking into account the possibility of repeat hepatectomy. For these reasons, in patients with multiple bilobar metastases, if complete resection is possible, the one-stage parenchymal-sparing hepatectomy should be favoured and should be considered as the gold-standard technique in patients with CRLM. Indeed, it has been reported that the parenchymal-sparing procedure, rather than a major hepatectomy, may increase the possibility of repeat hepatectomy in case of liver recurrence. However, in selected patients, TSH may be indicated. Indications for TSH include patients with multiple and bilateral CRLM in whom complete removal of all metastases is not feasible by a single 
hepatectomy because of insufficient future remnant liver.

Several studies have focused on the possibility of repeat hepatectomy in selected patients, also after major hepatectomy, and on the impact on overall survival. The rate of repeat hepatectomy may vary according to the expertise and to the surgical policy of the Center. The reported rates of repeat hepatectomy range from $8 \%$ to $35 \%$ (14). A recent Eastern paper by Takamoto et al. (14) reported a high rate of repeat hepatectomy ( $50 \%$ for all recurrences and $87 \%$ for liver only recurrences).

However, few studies evaluated the management of patients with liver recurrence following TSH $(12,15)$. A recent interesting paper by Imai et al. (15) analyzed the results of repeat surgery in one of the largest series of TSH for CRLM. In this study, among 139 patients with advanced bilateral CRLM scheduled for TSH between 1992 and 2012, 93 completed the TSH program and finally, a potentially curative surgery with resection of extrahepatic disease and of the primary tumor was achieved in 81 patients. Rate of recurrence in such patients was $76.5 \%(62$ patients). The overall rate of repeat surgery was $53.2 \%$ and reached $60.4 \%$ in patients with single-site recurrence.

The 5-year overall survival rate in patients who completed the TSH was $41.3 \%$. This result was similar to that published in other series $(11,12)$ and confirmed that the TSH strategy may add a survival benefit in selected patients. Recurrence rate in such patients was high (76.5\%) and the 5-year disease-free survival rate was low (8.9\%), however the 5 -year overall survival rate was similar to that following single liver resection (41.3\%). These results may be explained by the high rate of repeat surgery performed in this series $(53 \%)$, which had a significant impact on the long-term outcome of such patients. Indeed, the study showed that patients who underwent repeat surgery presented a significantly higher overall survival than those who did not. Moreover, at multivariate analysis, repeat surgery was an independent favorable prognostic factor. The authors showed that, although the disease-free survival was short (the time interval between second-stage hepatectomy and the first recurrence), the secondary disease-free survival (the time interval between second-stage hepatectomy and the first recurrence that could not be curatively treated) was longer, confirming the impact and benefit of repeat surgery.

The crucial issue analyzed in the study by Imai et al. (15) is the possibility of repeat liver resection following extended hepatectomy. Generally, repeat liver resection following major hepatectomy may be more difficult and less frequent. In the Eastern paper by Takamoto et al. (14) it has been showed that repeat liver resection was feasible and safe even if major hepatectomy was initially performed. Moreover, the type of first liver resection did not have any significant impact on the overall survival after repeat hepatectomy. Indeed, no significant difference in overall survival was found between patients who received repeat hepatectomy after major hepatectomy and those after minor hepatectomy.

The paper by Imai et al. (15) demonstrated that repeat hepatectomy was feasible even after one of the most aggressive approach such as TSH. The Paul Brousse experience (15), together with that from the MD Anderson Cancer Center (12), showed the results of the largest surgical series of TSH. The rates of repeat surgery following TSH in these two Centers were $53.2 \%$ and $37 \%$, respectively, comparable to those reported in other surgical series following minor liver resections. In other words, repeat hepatectomy shows a fundamental impact on longterm outcome in patients with recurrent CRLM and, as demonstrated for major hepatectomies by Prof. Makuuchi's series (14) and for TSH by Prof. Adam's series (15), the type of the first liver resection was not always correlated with the possibility of re-resection. The paper by Imai et al. (15) highlights that the possibility of repeat hepatectomy is strongly correlated with the aggressiveness of surgical management, which could be different according to the expertise and the policy of each Center.

Finally, as suggested in the paper by Imai et al. (15), this aggressive surgical management of recurrent disease may be applied also in patients with recurrence following ALPPS.

In conclusions, in case of extensive multiple bilateral CRLM, all the possible strategies (TSH, one-stage parenchymal-sparing hepatectomy and ALLPS) should be evaluated and accurately tailored to each patient. We do not have yet enough definitive data to classify one technique oncologically superior to each other. It should be highlighted that indication to perform TSH will not preclude the possibility of repeat hepatectomy. For these reasons, the choice should be based on the characteristics of the disease, of the patient and of the liver parenchyma, and on the safety of each procedure in each surgical center.

\section{Acknowledgments}

None.

\section{Footnote}

Conflicts of Interest: The author has no conflicts of interest to 
declare.

Ethical Statement: The author is accountable for all aspects of the work in ensuring that questions related to the accuracy or integrity of any part of the work are appropriately investigated and resolved.

\section{References}

1. Kopetz S, Chang GJ, Overman MJ, et al. Improved survival in metastatic colorectal cancer is associated with adoption of hepatic resection and improved chemotherapy. J Clin Oncol 2009;27:3677-83.

2. Ardito F, Panettieri E, Vellone $M$, et al. The impact of R1 resection for colorectal liver metastases on local recurrence and overall survival in the era of modern chemotherapy: an analysis of 1,428 resection areas. Surgery 2019;165:712-20.

3. de Haas RJ, Wicherts DA, Andreani P, et al. Impact of expanding criteria for resectability of colorectal metastases on short- and long-term outcomes after hepatic resection. Ann Surg 2011;253:1069-79.

4. Ardito F, Vellone M, Cassano A, et al. Chance of cure following liver resection for initially unresectable colorectal metastases: analysis of actual 5 -year survival. J Gastrointest Surg 2013;17:352-9.

5. Folprecht G, Gruenberger T, Bechstein W, et al. Survival of patients with initially unresectable colorectal liver metastases treated with FOLFOX/cetuximab or FOLFIRI/ cetuximab in a multidisciplinary concept (CELIM study). Ann Oncol 2014;25:1018-25.

6. Adam R, Laurent A, Azoulay D et al. Two-stage hepatectomy: a planned strategy to treat irresectable liver tumors. Ann Surg 2000;232:777-85.

7. Torzilli G, Procopio F, Botea F, et al. One-stage ultrasonographically guided hepatectomy for multiple bilobar colo- rectal metastases: a feasible and effective alternative to the 2-stage approach. Surgery 2009;146:60-71.

8. Schnitzbauer AA, Lang SA, Goessmann H, et al. Right portal vein ligation combined with in situ splitting induces rapid left lateral liver lobe hypertrophy enabling 2 -staged extended right hepatic resection in small-for-size settings. Ann Surg 2012;255:405-14.

9. Omichi K, Shindoh J, Cloyd JM, et al. Liver resection is justified for patients with bilateral multiple colorectal liver metastases: a propensity-score-matched analysis. Eur J Surg Oncol 2018;44:122-9.

10. de Jong MC, Pulitano C, Ribero D, et al. Rates and patterns of recurrence following curative intent surgery for colorectal liver metastasis: an international multiinstitutional analysis of 1669 patients. Ann Surg 2009;250:440-8.

11. Giuliante F, Ardito F, Ferrero A, et al. Tumor progression during preoperative chemotherapy predicts failure to complete 2-stage hepatectomy for colorectal liver metastases: results of an Italian multicenter analysis of 130 patients. J Am Coll Surg 2014;219:285-94.

12. Lillemoe HA, Kawaguchi Y, Passot G, et al. Surgical Resection for Recurrence After Two-Stage Hepatectomy for Colorectal Liver Metastases Is Feasible, Is Safe, and Improves Survival. J Gastrointest Surg 2019;23:84-92 .

13. Wicherts DA, de Haas RJ, Salloum C, et al. Repeat hepatectomy for recurrent colorectal metastases. Br J Surg 2013;100:808-18.

14. Takamoto T, Hashimoto T, Miyata A, et al. Repeat Hepatectomy After Major Hepatectomy for Colorectal Liver Metastases. J Gastrointest Surg 2019. [Epub ahead of print].

15. Imai K, Benitez CC, Allard MA, et al. Impact of Surgical Treatment for Recurrence After 2-Stage Hepatectomy for Colorectal Liver Metastases, on Patient Outcome. Ann Surg 2019;269:322-30.
Cite this article as: Ardito F. Possibility of repeat surgery for recurrence following two-stage hepatectomy for colorectal liver metastases: impact on patient outcome. Hepatobiliary Surg Nutr 2020;9(1):83-85. doi: 10.21037/hbsn.2019.09.08 